\title{
Aprendizaje e innovación: retos en las organizaciones del siglo XXI
}

\section{Learning and innovation: 21 st century challenges for organizations}

\author{
Félix Colina Ysea ${ }^{1}$ \\ José L. Albites Sanabria ${ }^{2}$ \\ Universidad Científica del Sur. Lima, Perú \\ Universidad Científica del Sur. Lima, Perú \\ fcolina@cientifica.edu.pe \\ jalbites@cientifica.edu.pe \\ Universidad Tecnológica del Perú, Lima, Perú ORCID 0000-0001-7688-6221 \\ C19271@utp.edu.pe \\ ORCID 0000-0002-6651-3509
}

Citar como: Colina, F. y Albites, J. (2020). Aprendizaje e innovación: retos en las organizaciones del siglo XXI. Desde el Sur, 12(1), pp. 167-176.

\section{RESUMEN}

Con las transformaciones generadas por la globalización -el mundo posmoderno, la era tecnológica, la incertidumbre, el caos-, las organizaciones han originado cambios transcendentales desde su visión paradigmática, como eje medular para afrontar las distintas realidades contextuales. Desde esta visión, el aprender a aprender en las organizaciones representa un bastión importante y estrategia de mejora continua en su talento humano, que debe instaurarse en toda la estructura organizativa de la empresa. Por lo tanto, el presente artículo de revisión tiene como objetivo general analizar la nueva concepción de las organizaciones basado en el aprender a aprender. Urgen cambios fundamentales en las organizaciones desde su talento

\footnotetext{
1 Doctor en Ciencias de la Educación. Magíster en Ciencias, con mención en Orientación de la Conducta. Licenciado en Educación en Lengua, con mención en Lengua, Literatura y Latín. Tiene 15 años de experiencia en docencia universitaria. Coach pedagógico y orientador en crecimiento personal. Investigador en el área de la educación y procesos psicológicos. Formador de profesionales integrales cónsonos a los principios humanos, valores sociales, mediante el desarrollo de competencias y la investigación como estrategia polivalente de saberes y proceso recurrente sobre la praxis.

2 Magíster en Ingeniería Biomédica de la Universidad de Brown (Providence, Rhode Island, Estados Unidos). La ingeniería biomédica se enfoca al desarrollo de interfaces cerebrocomputador. Mediante la extracción de señales neuronales en la corteza motora podemos predecir las intenciones de movimiento de una persona y utilizar esta señal en el control de computadores y prótesis robóticas. Esta tecnología busca restaurar la movilidad e independencia en personas con parálisis, las cuales, como producto de alguna enfermedad o accidente, perdieron la capacidad de controlar a voluntad una o más extremidades del cuerpo.
} 
humano, comprendiendo que los procesos de aprendizaje se logran desde las distintas experiencias vividas.

\section{PALABRAS CLAVE}

Organización, aprender a aprender, colaboradores, cambios, innovación

\section{ABSTRACT}

With the changes being generated by globalization, the postmodern world, the technological era, uncertainty and chaos, organizations have originated radical changes in their paradigmatic vision, modifying their core values in order to confront different contextual realities. In this context, the process of learning to learn within organizations constitutes an important bulwark and strategy for the ongoing improvement of human resources, the establishment of which is required throughout the organizational structure of a company. The general aim of this review article is to analyze the new learning to learn concept of organizations. Fundamental organizational changes are urgently needed at the level of human resources, through an understanding that learning processes are achieved from a multitude of different experiences.

\section{KEYWORDS}

Organization, learning to learn, collaborators, changes, innovation

\section{Introducción}

El mundo posmoderno enfrenta grandes cambios a nivel político, social, económico, tecnológico, culturales, entre otros, además de los cambios originados por la globalización, la posmodernidad, la incertidumbre, el caos, que motivan procesos de transformación en las organizaciones, en donde el aprender a aprender se convierte en el eje dinamizador de cambios instaurados desde el grupo de personas que la conforman.

En estos tiempos de cambios, las organizaciones deben asumir retos en su gestión estratégico-filosófica, centrados en enfoques humanísticos, lo que conlleva a formar al mejor talento humano para afrontar la incertidumbre, y genere el ganar ganar como estrategia en donde se empodere la organización, se beneficie a los colaboradores y se maximice la productividad. 
Bajo este contexto, las organizaciones deben asumir en su estructura medular el aprendizaje como proceso fundamental en todo el talento humano. Las decisiones y los niveles de cooperación de los colaboradores traen cambios en la cultura organizacional, e integran la visión filosófica y al trabajo colaborativo del talento humano. De esta forma, se logra una sinergia organizacional que establece mejora continua sobre la problemática detectada, y se asumen decisiones favorables a la productividad tanto de la empresa como de los trabajadores. Por lo tanto, el artículo tiene como objetivo analizar la nueva concepción de las organizaciones basado en el aprender a aprender.

\section{Metodología}

En la investigación se asumió la hermenéutica como proceso para analizar, comprender e interpretar los significados asociados a organizaciones que aprenden y la innovación en las organizaciones que aprenden. Se recurrió a la técnica de análisis del discurso para develar las categorías centrales en el estudio.

\section{Resultados}

\section{Organizaciones que aprenden a aprender}

De acuerdo con Fayol (1981), las organizaciones nacen para atender las necesidades de la sociedad. Se adaptan a los cambios generados por el mundo posmoderno mediante la creación de nuevas formas de aprendizaje y originan transformaciones en sus estructuras organizativas. Bajo esta perspectiva, se asume una nueva vertiente en las organizaciones desde la gestión del conocimiento como factor fundamental, que desarrolla y establece con rapidez la adquisición de competencias, lo que se traduce en la implementación de rediseños de los enfoques tradicionales de las organizaciones (Ayala, 2019). Por ejemplo, es necesario un viraje hacia lo humano, tecnológico, creativo, dinámico, a la generación de riqueza y, por lo tanto, a un nuevo enfoque en la gestión organizacional al que muchos autores han denominado la organización que aprende.

Para Bolívar (2000), las organizaciones que aprenden tienen instaurados procesos de reflexión, aprendizaje compartido, liderazgo transformacional, planificación organizacional centrada en las necesidades del talento humano y valoración de las acciones adquieren competencias (aprender a aprender), que implican transformación en los modelos mentales, además de la sinergia compartida. Estos axiomas constituyen el punto referencial en las organizaciones como procesos para valorar el conocimiento tácito y logran el conocimiento en su propio desarrollo. 
Para Colina y Martínez (2019), una organización que aprende es aqueIla que ha permitido el desarrollar, en todos los miembros que la conforman, sin importar cargo o nivel que ocupan en la estructura organizativa, capacidades continuas de adaptación y cambios. Esto quiere decir que buscan, a través del conocimiento, corregir los errores al implementar objetivos y políticas innovadoras para proporcionar soluciones diferentes, y establecer mejoras continuas en términos de efectividad.

Desde esta perspectiva, se hace necesario que los colaboradores de las organizaciones internalicen el aprendizaje organizacional, en donde se fomenten nuevas habilidades, se reafirmen competencias y destrezas, se intercambien experiencias, y se establezcan valores, como ejes multidimensionales en la comprensión de saberes desde el contexto organizacional, tal como lo reafirman Guanipa, Albites, Aldana y Colina (2019).

Entonces, las organizaciones que aprenden deben instaurar en su estructura organizativa que es menester promover la confianza en todo el talento humano, ideando espacios en donde se comparta conocimiento tácito y se convierta en experiencias de aprendizaje, es decir, fomentar el aprendizaje en equipo para que sea productivo, gestando el conocimiento explícito. Desde esta visión, se debe promover diferentes estilos de aprendizaje organizacional, en donde se intercambien experiencias, se establezca la comunicación asertiva, y se fomente la sinergia como proceso colaborativo, integrador y sistémico en la forma de pensar, percibir y generar ideas. De esta forma, se gesta una cultura de aprendizaje horizontal en donde todos aprenden de todos.

Para fomentar el aprendizaje como factor medular de las organizaciones que aprenden es necesario promover un ambiente de confianza, creatividad, flexibilidad, sentido de pertenencia, responsabilidad compartida e integración, pues al considerar estos elementos como vitales, se generan en todos los colaboradores actitudes de compromiso y aceptación al aprendizaje, lo que trae consigo cambios positivos como oportunidades de crecer.

En este sentido, el tomar a consideración el aprendizaje organizacional para el desarrollo de nuevas prácticas, actividades, tareas, herramientas y métodos de dirección permite el apuntalamiento de las organizaciones en el mercado globalizado, pues, cuando se da la oportunidad a los colaboradores de involucrarse en buscar alternativas de soluciones a problemas detectados, se genera mayor compromiso y pertinencia con la organización, y le permite alcanzar niveles más altos de innovación. Así, las personas que laboran en ellas expanden continuamente sus aptitudes y crean resultados asertivos, empoderamiento y valor a la organización. Además, se busca con ello que se cultiven nuevos esquemas de pensa- 
miento, avivando desde la libertad de las personas diferentes formas de aprender a aprender, para lograr el éxito de la gerencia y, por ende, el éxito de cada uno de sus colaboradores.

Desde esta perspectiva, una organización que no pone en práctica el aprendizaje continuo (aprender) quedará proscrita a un segundo plano, pues los cambios actuales engendran transformaciones fundamentales en la visión filosófico-estratégica de la organización, y tienen como proceso medular la formación perenne de los colaboradores. Por ello, combinar la práctica y el aprendizaje permite emerger un nuevo mundo de posibilidades para las organizaciones al contemplar la gestión del conocimiento dentro de las organizaciones como «sistemas que aprenden».

\section{La innovación en las organizaciones que aprenden}

La innovación se ha convertido en un factor esencial para las organizaciones del siglo XXI, lo que se refleja en todos sus procesos medulares e implica cambios trascendentales en visión paradigmática (Colina, 2019). Asimismo, la innovación es fundamental para evitar el fracaso de las organizaciones (Ayala, 2019). Por lo tanto, estudios en el área organizacional estiman esfuerzos al desarrollo de teorías, enfoques o tendencias que explican el resurgir de las organizaciones dentro de un ambiente de incertidumbre, generan estrategias de impacto en el talento humano y promueven el pensamiento creativo, reflexivo, crítico en cada uno de ellos, mediante nuevas formas de aprender desde la gestión del conocimiento.

En tal sentido, las organizaciones deben enfrentar los retos del mundo posmoderno atrapado por la incertidumbre, el caos, el encantado mundo de la globalización, los cambios generados por la tecnología de la información, las crisis económicas, los modelos sociales-culturales, tal como lo refiere Sanz (2014), plantean los diferentes desafíos condicionados en el mundo de las organizaciones.

Al respecto, las organizaciones deben converger con estos cambios, transformando las relaciones de producción, poder y experiencia, en las sociedades que se percibe están constituidas, pero además generando transformaciones y gestando contradicciones, crisis y modificaciones que dejan perpleja, vulnerable e incrédula a gran parte de la sociedad, lo cual hace obsoleta la era del industrialismo y forjan la época donde predomina la formación por encima del conocimiento, según Rodríguez y Colina (2016).

Desde esta perspectiva, el predominio de la razón instrumental trae consigo la reducción positivista de la idea de ciencia de hechos con la consiguiente indiferencia ante las cuestiones. Sin embargo, a nivel de las organizaciones todavía no se termina de arribar con paradigmas emergentes 
o centrados en las personas, que generen cambios sustanciales en la estructura organizativa de la organización.

Esta visión se sustenta en lo expresado por Descartes (2007), el ideal del conocimiento al servicio del hombre en aras de dominar a la naturaleza, la disociación entre naturaleza y cultura, es consecuencia de la cosmovisión propia de la sociedad industrial, y constituye las bases de los principios de ciencia y cientificidad fortalecidos en la modernidad, que tienen como importante pilar el pensamiento cartesiano.

Se comprende entonces que el racionalismo cartesiano es la efigie de un contexto que traspasa el mundo biofísico, al considerar que esta no puede concebirse sin lo biológico, pues la metamorfosis exige del hombre que se acople a su contexto natural, gracias a su capacidad de adaptarse y establecer vínculos sociales con otros. Desde esta visión, se asume que el hombre, al adaptarse a su contexto natural, también genera un proceso de cambios que impactan en la calidad de vida.

Es fácil comprender cómo el paradigma racionalista de la modernidad ha estado sumergido en una oscura crisis y, por tanto, es precisa su crítica; sin duda es un término que, junto con la incertidumbre, mejor define el presente histórico. Es así un tanto superlativo que autores como Lanni sostengan que «la razón crítica hace posible pensar en todo esto: modernidad, posmodernidad, pasado, presente, continuidad discontinuidad, realidad, simulacro, explicación, comprensión, micro, macro y meta no es un sueño, es una realidad» $(2007$, p. 125).

Bajo esta perspectiva, las organizaciones deben desaprender de los antiguos postulados gerenciales anclados en la corriente reduccionista, simplificador y mutilador de la multidimensionalidad de la condición humana, enmarcada en un paradigma que acude a lo científico para hacer ver que es la única forma de llegar a la verdad. Por lo tanto, el reto de las organizaciones, en sus diversas dimensiones, es aprender a desaprender, lo que conlleva a aprender a aprender dentro de una sociedad líquida que amerita el abordaje desde la multidimensionalidad del ser en el ser.

De esta forma, se avala con el discurrir de las relaciones humanas para la resignificación del saber, el hacer, el convivir, centrado en el ser; es decir, asumir los procesos personales, sociales, tecnológicos, medioambientales, económicos, deportivos, culturales, en formas entretejidas, juntas y religadas para la búsqueda, tanto del bienestar personal, la salud organizacional, el equilibrio de la sociedad. Esta aseveración se corresponde con lo planteado por Morín, Ciurana y Motta (2006, p. 54), cuando enuncian, al resaltar el paradigma de la complejidad, que: 
La complejidad es, efectivamente, el tejido de eventos, acciones, interacciones, cambios de direcciones, giros llamativos, retroacciones, determinaciones, azares, que constituyen nuestro mundo fenoménico. Así es que la complejidad en este ámbito de la gerencia se presenta con los rasgos perturbadores de la perplejidad, es decir, de lo enredado, lo inextricable, el desorden, la ambigüedad y la incertidumbre, lo magistral, pero también lo desconocido.

En el mundo entramado de la complejidad las acciones humanas constituyen sistemas de ejes medulares dinamizadores de las acciones dentro de las organizaciones, en donde las interacciones simbólicas del hombre determinan lo conocido y lo desconocido, dentro de un mundo de caos, crisis e incertidumbre. La complejidad plantea fenómenos en todos los contextos que rodean al ser, rodeados de distintas interacciones simbólicas que determina la cosmovisión del hombre con su propia visión de mundo. En este mismo orden de ideas, Najmanovich (2007) invita a desarrollar y afianzar el modo de pensar complejo, puesto que en todo ello es fundamental contextualizar la realidad, buscar la conexión de los fenómenos, construir el conocimiento con pertinencia y tener una actuación flexible en todos los planos de la vida.

Ello significa pensar en una gestión con personas desde la perspectiva de una sistemología interpretativa, pero también desde una antropoética humanizadora y complejizante. De esa manera, el planteamiento expresado anteriormente no se aleja de lo afirmado por Tünnermann (2008, p. 178), quien sostiene:

los cambios generados en las organizaciones presentan una realidad caracterizada por una altísima complejidad que amerita ser abordada con una visión holística. Las transformaciones que deberán producirse para lograr el progreso y desarrollo de la sociedad se fundamentan en el recurso organizacional más importante que es el conocimiento.

En tal sentido, el conocimiento se establece en constante movimiento, es decir, evoluciona con el pasar del tiempo, lo que supone cambios y transformaciones dentro de un mundo de complejidad, de incertidumbre, de caos, y exige cambios sustanciales en las estructuras sociales en las que hace vida el hombre. De esta realidad, las organizaciones empresariales deben asumir el cambio como proceso de mejora continua en cada una de las funciones establecidas, que promueven procesos de innovación y tienen como eje medular el aprendizaje continuo en su talento humano.

Es así como las organizaciones empresariales no son una excepción, debido a que han transitado la vía de objetivos institucionales, donde el desarrollo del conocimiento es una condición generada por las exigencias que hace una sociedad. Han transitado por proceso de globalización, 
caracterizados por cambios muy veloces, continuos y profundos, y que han permitido realizar procesos adecuados, innovadores y actualizaciones en términos de innovación. Esto garantiza una dinámica social que demanda de forma irremediable la consolidación del hombre como ser gregario.

\section{Conclusiones}

Los cambios generados por la globalización, la era tecnológica, la crisis en los sistemas económicos, políticos, culturales, sociales, han conllevando a que las organizaciones establezcan la reingeniería como un proceso de aprendizaje centrado en la participación de todos los colaboradores, bajo una visión sistémica, compartida y colaborativa, que permite el desarrollo de habilidades, destrezas y competencias desde el lugar de trabajo, y genera una nueva cultura organizacional basada en el aprendizaje continuo.

Desde esta visión, la innovación en las organizaciones que aprenden tiene retos a superar en su estructura organizativa, visión filosófica, gerencial y estratégica. Su eje medular es la formación continua de los colaboradores, debido a que en el aprender a aprender se cimientan las bases estratégicas y medulares para enrumbar a las organizaciones a estar a la vanguardia de los cambios del mundo posmoderno.

Entonces, la innovación en las organizaciones que aprenden se asume desde la forma creativa de selección, organización y utilización del talento humano y materiales, y conlleva a obtener resultados productivos tanto para los colaboradores como para la organización en sí. Este proceso circundante de complejidad origina cambios sustanciales en las organizaciones, e implica nuevas formas de comportamiento, estilos de aprendizaje y percepciones diferentes en el talento humano, en una conexión ineludible con la visión filosófica de la organización.

\section{Contribuciones}

Félix Colina Ysea ha participado en la recolección de la información, la revisión y la redacción del artículo.

José Albites Sanabria ha participado en la concepción, la recolección de información y la redacción del artículo.

\section{Fuentes de financiamiento}

Financiamiento no monetario.

\section{Conflicto de intereses}

Ninguno. 


\section{REFERENCIAS BIBLIOGRÁFICAS}

Ayala, Y. (2019). El método Lean Startup. Cómo crear empresas de éxito utilizando la innovación continua de Eric Ries. Emprende y Transforma, 1(1), pp. 83-88. Recuperado de https://doi.org/10.33829//emprendeytransforma-0101-2019-83-88

Bolívar, A. (2000). El liderazgo compartido según Peter Senge. En «Liderazgo y organizaciones que aprenden». III Congreso Internacional sobre Dirección de Centros Educativos. Bilbao: ICE de la Universidad de Deusto.

Colina, F. y Martínez, A. (2019). Una mirada crítica sobre los estilos de liderazgo en las organizaciones contemporáneas. Emprende y Transforma, 1(1), pp. 21-27. Recuperado de https://doi.org/10.33829/emprendeytransforma-0101-2019-21-27

Colina, F. (2019). La era de la perplejidad. Repensar el mundo que conocíamos por BBVA Open Mind (Barcelona, Penguin Random House Grupo Editorial, 2017). Emprende y Transforma, 1(1), pp. 93-96. Recuperado de https://doi.org/10.33829/emprendeytransforma-0101-2019-93-96

Descartes, R. (2007). El mecanismo y la complejidad. Ciudad de México: McGraw-Hill.

Fayol, H. (1981). Administración industrialy general. Buenos Aires: El Ateneo.

Guanipa Ramírez, L., Albites Sanabria, J., Aldana Zavala, J. y Colina Ysea, F. (2019). Educación para la ciudadanía y la democracia: el equilibrio del poder. Iustitia Socialis, 4(6), pp. 71-89. Recuperado de http://dx.doi. org/10.35381/racji.v4i6.290

Lanni, O. (2007). La sociedad global. Ciudad de México: Siglo XXI.

Marquardt, M. (1996). Building the Learning Organization. Nueva York: McGraw-Hill.

Morin, E., Ciurana, E. y Motta, R. (2006). Educar en la era planetaria. Valladolid: Universidad de Valladolid.

Najmanovich, D. (2007). La complejidad en las organizaciones. Buenos Aires: Ceace.

Rodríguez Acasio, F. y Colina Ysea, F. (2016). La investigación desde la gestión del conocimiento en el contexto de las universidades nacionales experimentales. Revista Arbitrada Interdisciplinaria Koinonía, 1(1), pp. 88-100. Recuperado de http://fundacionkoinonia.com.ve/ojs/index.php/revistakoinonia/article/view/17/10

Sanz, G. (2003). La economía informal a finales del siglo XX: viejos dilemas y nuevos debates. En C. Bueno y E. Aguilar (Coords.), Las expresiones 
locales de la globalización: México y España (pp. 219-240). Ciudad de México: Ciesas.

Tunnerman, C. (2008). La universidad necesita transformarse. Medellín: Universidad de Medellín.

Recibido: 9/3/2019

Aceptado: 22/7/2019 